\title{
Sums of three squareful numbers
}

\author{
T.D. Browning and K. Van Valckenborgh
}

\begin{abstract}
We investigate the frequency of positive squareful numbers $x, y, z \leqslant B$ for which $x+y=z$ and present a conjecture concerning its asymptotic behaviour.
\end{abstract}

Mathematics Subject Classification (2010). 11D45 (11P05, 14G05).

\section{Introduction}

In this paper we examine the quantitative arithmetic of integral points on certain Campana orbifolds, following the discussions of Abramovich [1], Campana [3] and Poonen [10]. Given rational points $p_{i}=r_{i} / s_{i} \in \mathbf{P}^{1}(\mathbf{Q})$ with integer multiplicities $m_{i} \geqslant 2$, for $1 \leqslant i \leqslant n$, we define the divisor $\Delta=\sum_{i}\left(1-\frac{1}{m_{i}}\right)\left[p_{i}\right]$. The pair $\left(\mathbf{P}^{1}, \Delta\right)$ defines an orbifold curve in the sense of Campana and has associated Euler characteristic

$$
\chi=\chi\left(\mathbf{P}^{1}\right)-\operatorname{deg} \Delta=2-n+\frac{1}{m_{1}}+\cdots+\frac{1}{m_{n}} .
$$

A point $r / s \in \mathbf{P}^{1}(\mathbf{Q})$ is said to be integral if $r s_{i}-s r_{i}$ is $m_{i}$-powerful for $1 \leqslant i \leqslant n$. Here we recall that an integer $k$ is said to be $m$-powerful if $p^{m} \mid k$ whenever $p$ is a prime divisor of $k$. We will focus our attention here upon the orbifold $\left(\mathbf{P}^{1}, \Delta\right)$ associated to the divisor

$$
\Delta=\left(1-\frac{1}{m}\right)[0]+\left(1-\frac{1}{m}\right)[1]+\left(1-\frac{1}{m}\right)[\infty],
$$

with Euler characteristic $\chi=-1+\frac{3}{m}$. The density of integral points on $\left(\mathbf{P}^{1}, \Delta\right)$ with height at most $B$ is captured by the counting function

$$
N_{m-1}(B)=\#\left\{(x, y, z) \in \mathbf{N}_{\text {prim }}^{3}: x+y=z, x, y, z \leqslant B, x, y, z m \text {-powerful }\right\},
$$

where $\mathbf{N}$ denotes the set of positive integers and $\mathbf{N}_{\text {prim }}^{3}$ denotes the set of primitive vectors in $\mathbf{N}^{3}$.

An old result of Erdős and Szekeres [4] shows that the number of $m$-powerful integers up to $x$ is $c_{m} x^{\frac{1}{m}}+O\left(x^{\frac{1}{m+1}}\right)$, for a certain constant $c_{m}>0$. This leads to a basic trichotomy: we expect only finitely many integral points when $\chi<0$, we expect $N_{m-1}(B)$ to grow at worst logarithmically in $B$ when $\chi=0$ and we expect $N_{m-1}(B)$ to have order $B^{\chi}$ when $\chi>0$. When $m=3$ work of Nitaj [7] shows that $N_{2}(B) \gg \log B$. Our goal in this paper is to provide evidence in support of the expected order $B^{\frac{1}{2}}$ of $N_{1}(B)$ when $m=2$.

Conjecture 1. We have

$$
N_{1}(B)=c B^{\frac{1}{2}}(1+o(1))
$$

as $B \rightarrow \infty$, with $c=2.68 \ldots$

The explicit conjectured value of $c$ is too complicated to record here, but may be found in (13) and (14). Our expression for $c$ involves an infinite sum which converges very slowly, thereby making it difficult to evaluate numerically to high accuracy.

We may test Conjecture 1 by naively listing all squareful numbers up to $B$, and then subsequently sorting them into triples $(x, y, z)$ that are counted by $N_{1}(B)$. More precisely, the algorithm 


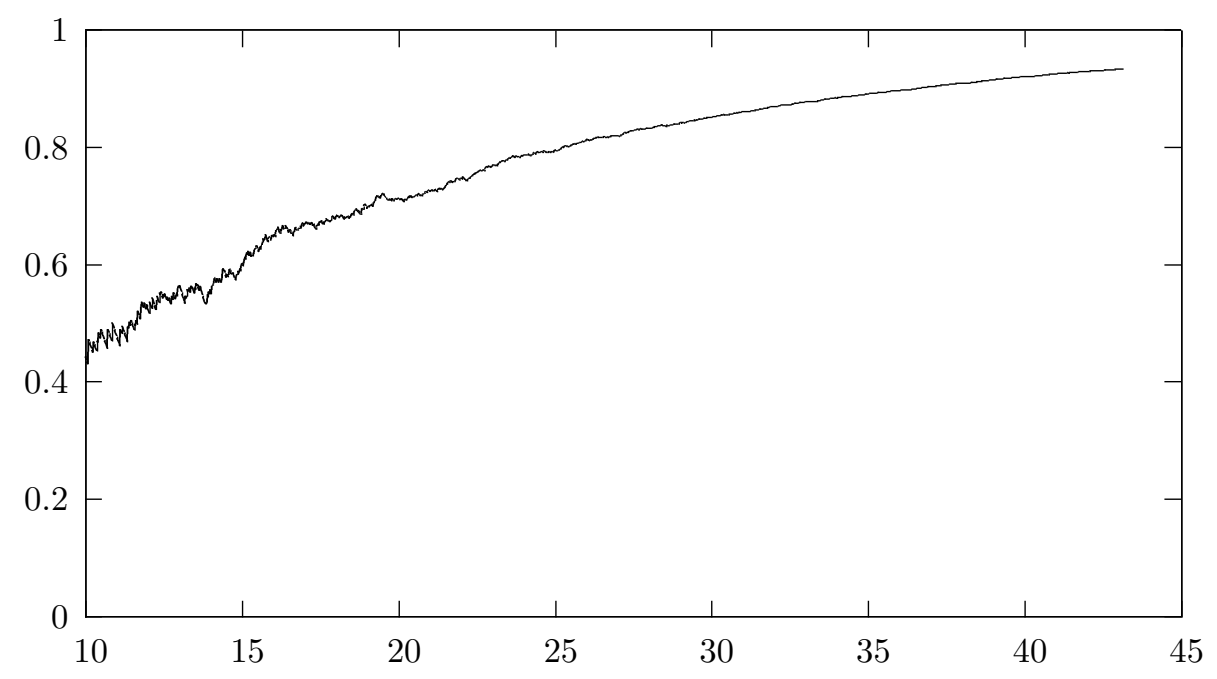

Figure 1. Values of $N_{1}(B) /\left(c B^{\frac{1}{2}}\right)$

loops through all squareful numbers $z$ in increasing order, and for each $z$, it runs over squareful $x \in[z / 2, z]$ and uses the list to verify whether or not $y=z-x$ is squareful. If it is, we verify whether $\operatorname{gcd}(x, y)=1$ and eventually print the two corresponding points $(x, y, z)$ and $(y, x, z)$. The inner code of the two loops is repeated $O\left(s^{2}\right)$ times, where $s$ is the number of squarefuls involved, so that the total complexity is $O(B)$. For $B=10^{13}$ the compilation of the list took less than 2 minutes on an Intel Core 2 Duo E8400 running at $3 \mathrm{GHz}$, resulting in 6840384 squareful numbers overall. The sorting algorithm required a computing time of 5587.5 minutes. In Figure 1 the values of $N_{1}(B) /\left(c B^{\frac{1}{2}}\right)$ are plotted for $B$ up to $10^{13}$, where the horizontal axis runs over values of $\log _{2} B$. In Table 1 we present some explicit numerical data, including the determination of the quotient $N_{1}(B) /\left(c B^{\frac{1}{2}}\right)$ for large values of $B$.

\begin{tabular}{|l|l|l|}
\hline$B$ & $N_{1}(B)$ & $N_{1}(B) /\left(c B^{\frac{1}{2}}\right)$, \\
\hline $10^{7}$ & 6562 & 0.774 \\
$10^{8}$ & 21920 & 0.818 \\
$10^{9}$ & 72124 & 0.851 \\
$10^{10}$ & 235168 & 0.877 \\
$10^{11}$ & 762580 & 0.900 \\
$10^{12}$ & 2465044 & 0.920 \\
$10^{13}$ & 7914884 & 0.934 \\
\hline
\end{tabular}

TABLE 1

Any positive squareful integer $k$ can be written uniquely as $k=x^{2} y^{3}$, with $x, y \in \mathbf{N}$ and $y$ square-free. Using this description we have

$$
N_{1}(B)=\sum_{\mathbf{y} \in \mathbf{N}^{3}} \mu^{2}\left(y_{0} y_{1} y_{2}\right) \#\left\{\mathbf{x} \in \mathbf{N}^{3} \cap C_{\mathbf{y}}: \begin{array}{l}
\operatorname{gcd}\left(x_{0} y_{0}, x_{1} y_{1}, x_{2} y_{2}\right)=1, \\
x_{0}^{2} y_{0}^{3}, x_{1}^{2} y_{1}^{3}, x_{2}^{2} y_{2}^{3} \leqslant B
\end{array}\right\},
$$

where $\mu$ is the Möbius function and $C_{\mathbf{y}}$ denotes the conic $x_{0}^{2} y_{0}^{3}+x_{1}^{2} y_{1}^{3}=x_{2}^{2} y_{2}^{3}$. One is naturally led to analyse $N_{1}(B)$ by counting points on each conic and then summing the contribution over the $\mathbf{y}$. This is the point of view adopted by the second author [11], where the structure of the orbifold $\left(\mathbf{P}^{1}, \Delta\right)$ is generalised to a higher-dimensional analogue $\left(\mathbf{P}^{n-1}, \Delta\right)$, corresponding to a hyperplane of squareful numbers. An asymptotic formula of the expected order of magnitude is then obtained when there are $n+1 \geqslant 5$ terms present in the hyperplane. In addition to this [11] 
contains an interpretation of the leading constant in terms of local densities for the underlying quadric. We will revisit this discussion in $\S 2$ in order to justify the numerical value of the constant in Conjecture 1.

Ignoring all but the term with $\mathbf{y}=(1,1,1)$ in $(1)$, one readily arrives at the lower bound $N_{1}(B) \gg B^{\frac{1}{2}}$, via the familiar parametrisation for Pythagorean triples. Building on this observation suitably, we will sketch a proof of the following result in $\S 3$.

Theorem 1. We have $N_{1}(B) \geqslant c B^{\frac{1}{2}}(1+o(1))$, where $c$ is the constant in Conjecture 1 .

The problem of producing an upper bound of the expected order of magnitude is much more challenging. In $\S 4$ we shall establish the following estimate.

Theorem 2. We have $N_{1}(B)=O\left(B^{\frac{3}{5}} \log ^{12} B\right)$.

With more work it ought to be possible to remove the factor involving $\log B$ from Theorem 2 . The proof of Theorem 2 involves two estimates. The first is based on fixing the $\mathbf{y}$ and counting points on the conic $C_{\mathbf{y}}$, uniformly in the coefficients. The second involves switching the rôles of $\mathbf{y}$ and $\mathbf{x}$, viewing the equation as a family of plane cubics instead. For both of these the determinant method of Heath-Brown [6] is a key tool. The same argument has been observed by a number of mathematicians, including Valentin Blomer in private communication with the first author. In order to improve the exponent of $B$ in Theorem 2 one requires a new means of treating the contribution from $\mathbf{x}, \mathbf{y}$ for which each $x_{i}$ and $y_{i}$ has order of magnitude $B^{\frac{1}{5}}$. It would be desirable, for example, to have better control over the $\mathbf{y}$ which produce conics $C_{\mathbf{y}}$ containing at least one rational point of small height.

Acknowledgements. The authors are grateful to Emmanuel Peyre and the anonymous referee for useful comments and to Hendrik Hubrechts for help with preparing the numerical evidence. While working on this paper the first author was supported by EPSRC grant number EP/E053262/1.

\section{The constant}

Recall the expression for $N_{1}(B)$ in $(1)$, in which $C_{\mathbf{y}}$ denotes the conic

$$
x_{0}^{2} y_{0}^{3}+x_{1}^{2} y_{1}^{3}=x_{2}^{2} y_{2}^{3},
$$

for given $\mathbf{y}=\left(y_{0}, y_{1}, y_{2}\right) \in \mathbf{N}^{3}$. Let $H_{\mathbf{y}}: C_{\mathbf{y}}(\mathbf{Q}) \rightarrow \mathbf{R}_{\geqslant 0}$ denote the height function

$$
\left[x_{0}, x_{1}, x_{2}\right] \mapsto \max \left\{\left|x_{0}^{2} y_{0}^{3}\right|,\left|x_{1}^{2} y_{1}^{3}\right|,\left|x_{2}^{2} y_{2}^{3}\right|\right\}^{\frac{1}{2}},
$$

if $x_{0}, x_{1}, x_{2} \in \mathbf{Z}$ satisfy $\operatorname{gcd}\left(x_{0}, x_{1}, x_{2}\right)=1$. On noting that $\mathbf{x}$ and $\mathbf{- x}$ represent the same point in $\mathbf{P}^{2}$ we easily infer that $N_{1}(B)$ is approximated by the sum

$$
\frac{1}{4} \sum_{\mathbf{y} \in \mathbf{N}^{3}} \mu^{2}\left(y_{0} y_{1} y_{2}\right) \#\left\{x \in C_{\mathbf{y}}(\mathbf{Q}): H_{\mathbf{y}}(x) \leqslant B^{\frac{1}{2}}, \operatorname{gcd}\left(x_{0} y_{0}, x_{1} y_{1}, x_{2} y_{2}\right)=1\right\} .
$$

Following the framework developed by the second author $[11, \S 5]$, we are therefore led to take the value

$$
c=\frac{1}{4} \sum_{\mathbf{y} \in \mathbf{N}^{3}} \mu^{2}\left(y_{0} y_{1} y_{2}\right) c_{H_{\mathbf{y}}}\left(C_{\mathbf{y}}\left(\mathbb{A}_{\mathbf{Q}}\right)^{+}\right),
$$

in Conjecture 1. Here, if $C_{\mathbf{y}}\left(\mathbb{A}_{\mathbf{Q}}\right)^{+}$denotes the open subset of the adelic space $C_{\mathbf{y}}\left(\mathbb{A}_{\mathbf{Q}}\right)$ carved out by the condition $\min _{0 \leqslant i \leqslant 2}\left\{v_{p}\left(x_{i, p} y_{i}\right)\right\}=0$ for each prime $p$, then $c_{H_{\mathbf{y}}}\left(C_{\mathbf{y}}\left(\mathbb{A}_{\mathbf{Q}}\right)^{+}\right)$is a special case of the constant conjecturally introduced by Peyre [8, Définition 2.5] in the broader context of Fano varieties. In particular it follows that

$$
c_{H_{\mathbf{y}}}\left(C_{\mathbf{y}}\left(\mathbb{A}_{\mathbf{Q}}\right)^{+}\right)=\alpha\left(C_{\mathbf{y}}\right) \boldsymbol{\omega}_{H_{\mathbf{y}}}\left(C_{\mathbf{y}}\left(\mathbb{A}_{\mathbf{Q}}\right)^{+}\right),
$$

where $\boldsymbol{\omega}_{H_{\mathbf{y}}}\left(C_{\mathbf{y}}\left(\mathbb{A}_{\mathbf{Q}}\right)^{+}\right)$denotes the Tamagawa measure of $C_{\mathbf{y}}\left(\mathbb{A}_{\mathbf{Q}}\right)^{+}$associated to the height $H_{\mathbf{y}}$ and $\alpha\left(C_{\mathbf{y}}\right)$ is the volume of a certain polytope contained in the cone of effective divisors. 
Let $\mathbf{y} \in \mathbf{N}^{3}$ with $\mu^{2}\left(y_{0} y_{1} y_{2}\right)=1$. In the present setting we have $\operatorname{Pic}\left(C_{\mathbf{y}}\right) \cong \mathbf{Z}$ and one finds, using [8, Définition 2.4], that

$$
\alpha\left(C_{\mathbf{y}}\right)=\frac{1}{2}
$$

In [11], wherein non-singular quadrics in $\mathbf{P}^{n}$ feature for $n \geqslant 4$, it is worth highlighting that the corresponding value of the constant is found to be $\frac{1}{n-1}$ using the Lefschetz hyperplane theorem. This is no longer true when considering conics in $\mathbf{P}^{2}$, since the anticanonical divisor is not a generator for the Picard group.

Turning to the Tamagawa constant we let $S=\{\infty, 2\} \cup\left\{p \mid y_{0} y_{1} y_{2}\right\}$, a finite set of places. The Tamagawa measure on $C_{\mathbf{y}}\left(\mathbb{A}_{\mathbf{Q}}\right)$ associated to the height function $H_{\mathbf{y}}$ is given by

$$
\boldsymbol{\omega}_{H_{\mathbf{y}}}=\lim _{s \rightarrow 1}(s-1) L_{S}\left(s, \operatorname{Pic}\left(\overline{C_{\mathbf{y}}}\right)\right) \prod_{v \in \operatorname{Val}(\mathbf{Q})} \lambda_{v}^{-1} \boldsymbol{\omega}_{H_{\mathbf{y}}, v},
$$

where

$$
\lambda_{v}= \begin{cases}\left(1-\frac{1}{p}\right)^{-1}, & \text { if } v \in \operatorname{Val}(\mathbf{Q})-S \\ 1, & \text { otherwise }\end{cases}
$$

and

$$
L_{S}\left(s, \operatorname{Pic}\left(\overline{C_{\mathbf{y}}}\right)\right)=\prod_{v \in \operatorname{Val}(\mathbf{Q})-S}\left(1-\frac{1}{p^{s}}\right)^{-1}=\zeta(s) \prod_{p \mid 2 y_{0} y_{1} y_{2}}\left(1-\frac{1}{p^{s}}\right)
$$

Hence

$$
\lim _{s \rightarrow 1}(s-1) L_{S}\left(s, \operatorname{Pic}\left(\overline{C_{\mathbf{y}}}\right)\right)=\prod_{p \mid 2 y_{0} y_{1} y_{2}}\left(1-\frac{1}{p}\right)
$$

In the next few sections, we will calculate the $v$-adic densities at the different places.

\subsection{Density at the good places}

Let $p$ be a prime such that $p \nmid 2 y_{0} y_{1} y_{2}$. Recall that $C_{\mathbf{y}}\left(\mathbf{Q}_{p}\right)^{+}$is defined as the subset of points $\left[x_{0, p}, x_{1, p}, x_{2, p}\right] \in C_{\mathbf{y}}\left(\mathbf{Q}_{p}\right)$, with $x_{i, p} \in \mathbf{Z}_{p}$ and $\min _{0 \leqslant i \leqslant 2}\left\{v_{p}\left(x_{i, p}\right)\right\}=0$, for which

$$
\min _{0 \leqslant i \leqslant 2}\left\{v_{p}\left(x_{i, p} y_{i}\right)\right\}=0 .
$$

Since $p \nmid y_{0} y_{1} y_{2}$ this latter condition is automatically satisfied, whence $C_{\mathbf{y}}\left(\mathbf{Q}_{p}\right)^{+}=C_{\mathbf{y}}\left(\mathbf{Q}_{p}\right)$. By Lemmas 3.2 and 3.4 in [9] and [8, Lemme 5.4.6], we have

$$
\boldsymbol{\omega}_{H_{\mathbf{y}}, p}\left(C_{\mathbf{y}}\left(\mathbf{Q}_{p}\right)\right)=\frac{\# C_{\mathbf{y}}\left(\mathbf{F}_{p}\right)}{p} .
$$

Since $C_{\mathbf{y}}\left(\mathbf{F}_{p}\right) \neq \emptyset$ by Chevalley-Warning, we deduce that $\# C_{\mathbf{y}}\left(\mathbf{F}_{p}\right)=\# \mathbf{P}^{1}\left(\mathbf{F}_{p}\right)=p+1$. This implies that for the good places we have

$$
\begin{aligned}
\prod_{v \in \operatorname{Val}(\mathbf{Q})-S} \lambda_{v}^{-1} \boldsymbol{\omega}_{H_{\mathbf{y}}, v}\left(C_{\mathbf{y}}\left(\mathbf{Q}_{v}\right)^{+}\right) & =\prod_{p \nmid 2 y_{0} y_{1} y_{2}}\left(1-\frac{1}{p}\right)\left(1+\frac{1}{p}\right) \\
& =\frac{8}{\pi^{2}} \cdot \prod_{\substack{p \mid y_{0} y_{1} y_{2} \\
p>2}}\left(1-\frac{1}{p^{2}}\right)^{-1}
\end{aligned}
$$

since $\prod_{p>2}\left(1-\frac{1}{p^{2}}\right)=\frac{4}{3} \cdot \frac{6}{\pi^{2}}=\frac{8}{\pi^{2}}$. 


\subsection{Density at the bad places}

We now suppose that $p$ is a prime divisor of $2 y_{0} y_{1} y_{2}$. In this case, when considering $C_{\mathbf{y}}\left(\mathbf{Q}_{p}\right)^{+}$, the condition (9) will no longer be satisfied trivially. Let

$$
N_{\mathbf{y}}^{*}\left(p^{r}\right)=\#\left\{\mathbf{x} \in\left(\mathbf{Z} / p^{r} \mathbf{Z}\right)^{3}-\left(p \mathbf{Z} / p^{r} \mathbf{Z}\right)^{3}: \begin{array}{l}
y_{0}^{3} x_{0}^{2}+y_{1}^{3} x_{1}^{2} \equiv y_{2}^{3} x_{2}^{2}\left(\bmod p^{r}\right), \\
\min _{0 \leqslant i \leqslant 2}\left\{v_{p}\left(x_{i} y_{i}\right)\right\}=0
\end{array}\right\} .
$$

Using Lemmas 3.2 and 3.4 in [9] and [8, Lemme 5.4.6], we deduce the existence of $r_{0} \in \mathbf{N}$ such that

$$
\boldsymbol{\omega}_{H_{\mathbf{y}}, p}\left(C_{\mathbf{y}}\left(\mathbf{Q}_{p}\right)^{+}\right)=\left(1-\frac{1}{p}\right)^{-1} \cdot \frac{N_{\mathbf{y}}^{*}\left(p^{r}\right)}{p^{2 r}},
$$

for each $r \geqslant r_{0}$. The following pair of results are concerned with the calculation of $N_{\mathbf{y}}^{*}\left(p^{r}\right)$ for primes $p \mid 2 y_{0} y_{1} y_{2}$.

Lemma 1. If $p \mid y_{0} y_{1} y_{2}$ and $p>2$, we have

$$
\frac{N_{\mathbf{y}}^{*}\left(p^{r}\right)}{p^{2 r}}=\left(1-\frac{1}{p}\right) \times \begin{cases}\left(1+\left(\frac{y_{1} y_{2}}{p}\right)\right), & \text { if } p \mid y_{0}, \\ \left.1+\left(\frac{y_{0} y_{2}}{p}\right)\right), & \text { if } p \mid y_{1}, \\ \left(1+\left(\frac{-y_{0} y_{1}}{p}\right)\right), & \text { if } p \mid y_{2} .\end{cases}
$$

Proof. Suppose, for example, that $p$ divides $y_{0}$. In this case $p \nmid y_{1} y_{2}$. Modulo $p$ we obtain the equation $y_{1}^{3} x_{1}^{2} \equiv y_{2}^{3} x_{2}^{2}(\bmod p)$. If $y_{1}^{-3} y_{2}^{3}$ is a square modulo $p$, then we can choose $x_{2}$ arbitrarily in $\mathbf{F}_{p}^{\times}$and for each choice of $x_{2}$ there are two solutions for $x_{1}$. It follows that there are $2 p(p-1)$ solutions modulo $p$ in this case. If $y_{1}^{-3} y_{2}^{3}$ is not a square modulo $p$, then there are no solutions. We conclude that $N_{\mathbf{y}}^{*}(p)=\left(1+\left(\frac{y_{1} y_{2}}{p}\right)\right) p(1-p)$. Using Hensel's lemma we deduce that $N_{\mathbf{y}}^{*}\left(p^{r}\right)$ is equal to $p^{2(r-1)}\left(1+\left(\frac{y_{1} y_{2}}{p}\right)\right) p(1-p)$ for each $r \geqslant 1$, which thereby completes the proof.

Lemma 2. If $r \geqslant 3$, we have

$$
\frac{N_{\mathbf{y}}^{*}\left(2^{r}\right)}{2^{2 r}}=\left\{\begin{array}{l}
1, \quad \text { if } 2 \nmid y_{0} y_{1} y_{2} \text { and } \neg\left\{y_{0} \equiv y_{1} \equiv-y_{2}(\bmod 4)\right\} \\
2, \quad \text { if } 2 \mid y_{0} \text { and } y_{1} \equiv y_{2}(\bmod 8), \\
2, \quad \text { if } 2 \mid y_{1} \text { and } y_{0} \equiv y_{2}(\bmod 8), \\
2, \quad \text { if } 2 \mid y_{2} \text { and } y_{0} \equiv-y_{1}(\bmod 8), \\
0, \quad \text { otherwise. }
\end{array}\right.
$$

Proof. This follows from direct calculation for the case $r=3$. The formula for $r>3$ follows from Hensel's lemma.

\subsection{Density at the infinite place}

It remains to consider the infinite place $v=\infty$. Let

$$
D_{1}=\left\{\left(y_{0}^{3} x_{0}^{2}, y_{1}^{3} x_{1}^{2}, y_{2}^{3} x_{2}^{2}\right) \in(\mathbf{R} \cap[-1,1])^{3}: y_{0}^{3} x_{0}^{2}+y_{1}^{3} x_{1}^{2}=y_{2}^{3} x_{2}^{2}\right\} .
$$

Using [8, Lemme 5.4.7], we obtain

$$
\boldsymbol{\omega}_{H_{\mathbf{y}}, \infty}\left(C_{\mathbf{y}}(\mathbf{R})^{+}\right)=\frac{1}{2} \cdot \int_{D_{1}} \boldsymbol{\omega}_{L, \infty},
$$

where

$$
\boldsymbol{\omega}_{L, \infty}=\frac{\mathrm{d} x_{0} \mathrm{~d} x_{1}}{2 y_{2}^{\frac{3}{2}} \sqrt{y_{0}^{3} x_{0}^{2}+y_{1}^{3} x_{1}^{2}}}
$$

is the Leray form. Let $D_{2}=\left\{\left(x_{0}, x_{1}\right) \in(\mathbf{R} \cap[-1,1])^{2}: x_{0}^{2}+x_{1}^{2} \leqslant 1\right\}$. Then it follows that

$$
\begin{aligned}
\boldsymbol{\omega}_{H_{\mathbf{y}}, \infty}\left(C_{\mathbf{y}}(\mathbf{R})^{+}\right) & =\frac{1}{2} \cdot \frac{1}{\left(y_{0} y_{1} y_{2}\right)^{\frac{3}{2}}} \int_{D_{2}} \frac{1}{\sqrt{x_{0}^{2}+x_{1}^{2}}} \mathrm{~d} x_{0} \mathrm{~d} x_{1} \\
& =\frac{\pi}{\left(y_{0} y_{1} y_{2}\right)^{\frac{3}{2}}} .
\end{aligned}
$$




\subsection{Conclusion}

Recall the definition (6) of the Tamagawa measure, in which the convergence factors are given by (7). Combining (8), (10), (11) with Lemma 1 and (12) we deduce that $\boldsymbol{\omega}_{H_{\mathbf{y}}}\left(C_{\mathbf{y}}\left(\mathbb{A}_{\mathbf{Q}}\right)^{+}\right)$is equal to

$$
\frac{1}{\left(y_{0} y_{1} y_{2}\right)^{\frac{3}{2}}} \cdot \frac{8}{\pi} \cdot \sigma_{2, \mathbf{y}} \cdot \prod_{\substack{p \mid y_{0} \\ p>2}} \frac{\left(1+\left(\frac{y_{1} y_{2}}{p}\right)\right)}{\left(1+\frac{1}{p}\right)} \cdot \prod_{\substack{p \mid y_{1} \\ p>2}} \frac{\left(1+\left(\frac{y_{0} y_{2}}{p}\right)\right)}{\left(1+\frac{1}{p}\right)} \cdot \prod_{\substack{p \mid y_{2} \\ p>2}} \frac{\left(1+\left(\frac{-y_{0} y_{1}}{p}\right)\right)}{\left(1+\frac{1}{p}\right)},
$$

where $\sigma_{2, \mathbf{y}}=\lim _{r \rightarrow \infty} 2^{-2 r} N_{\mathbf{y}}^{*}\left(2^{r}\right)$ is given by Lemma 2 . Substituting this into the definition of the conjectural constant (4), and combining it with (5), we deduce from (3) that

$$
\begin{aligned}
c=\frac{1}{\pi} & \cdot \sum_{\mathbf{y} \in \mathbf{N}^{3}} \frac{\mu^{2}\left(y_{0} y_{1} y_{2}\right)}{\left(y_{0} y_{1} y_{2}\right)^{\frac{3}{2}}} \cdot \sigma_{2, \mathbf{y}} \\
& \times \prod_{\substack{p \mid y_{0} \\
p>2}} \frac{\left(1+\left(\frac{y_{1} y_{2}}{p}\right)\right)}{\left(1+\frac{1}{p}\right)} \cdot \prod_{\substack{p \mid y_{1} \\
p>2}} \frac{\left(1+\left(\frac{y_{0} y_{2}}{p}\right)\right)}{\left(1+\frac{1}{p}\right)} \cdot \prod_{\substack{p \mid y_{2} \\
p>2}} \frac{\left(1+\left(\frac{-y_{0} y_{1}}{p}\right)\right)}{\left(1+\frac{1}{p}\right)} .
\end{aligned}
$$

In the remainder of this section we shall attempt to simplify this expression, in order to facilitate its numerical evaluation. Writing $S$ for the set of $\mathbf{y} \in \mathbf{N}^{3}$ for which $\mu^{2}\left(y_{0} y_{1} y_{2}\right)=1$, we can partition $S$ into subsets

$$
S_{-1}=\left\{\mathbf{y} \in S: 2 \nmid y_{0} y_{1} y_{2}\right\}, \quad S_{i}=\left\{\mathbf{y} \in S: 2 \mid y_{i}\right\},
$$

for $0 \leqslant i \leqslant 2$. We then split (13) into sums $c_{i}$ over $S_{i}$, for each $-1 \leqslant i \leqslant 2$. To streamline the notation, we define

$$
\gamma(n)=\prod_{p \mid n}\left(1+\frac{1}{p}\right)^{-1}
$$

and, for $a, b \in \mathbf{N}$ with $a, b$ squarefree and $b>1$ odd, we set $\left(\frac{a}{b}\right)_{*}=1$ if and only if $\left(\frac{a}{p}\right)=1$ for each $p \mid b$, with the convention that $\left(\frac{a}{1}\right)_{*}=1$.

We begin by examining $c_{-1}$, in which case $y_{0}, y_{1}$ and $y_{2}$ are all odd. We get

$$
\begin{aligned}
c_{-1}= & \frac{1}{\pi} \cdot \sum_{\substack{\mathbf{y} \in S_{-1} \\
\neg\left\{y_{0} \equiv y_{1} \equiv-y_{2}(\bmod 4)\right\}}} \frac{\gamma\left(y_{0} y_{1} y_{2}\right)}{\left(y_{0} y_{1} y_{2}\right)^{\frac{3}{2}}} \\
& \times \prod_{p \mid y_{0}}\left(1+\left(\frac{y_{1} y_{2}}{p}\right)\right) \cdot \prod_{p \mid y_{1}}\left(1+\left(\frac{y_{0} y_{2}}{p}\right)\right) \cdot \prod_{p \mid y_{2}}\left(1+\left(\frac{-y_{0} y_{1}}{p}\right)\right) .
\end{aligned}
$$

Substituting $d=y_{0} y_{1} y_{2}$, we obtain

$$
c_{-1}=\frac{1}{\pi} \cdot \sum_{\substack{d=1 \\ 2 \nmid d}}^{\infty} \frac{\mu^{2}(d) \cdot \gamma(d) \cdot 2^{\omega(d)}}{d^{\frac{3}{2}}} \cdot \Delta_{-1}(d),
$$

where $\omega(d)$ denotes the number of distinct prime divisors of $d$ and

$$
\Delta_{-1}(d)=\#\left\{\begin{array}{ll}
y_{0} y_{1} y_{2}=d: & \neg\left\{y_{0} \equiv y_{1} \equiv-y_{2}(\bmod 4)\right\} \\
\left(\frac{y_{1} y_{2}}{y_{0}}\right)_{*}=\left(\frac{y_{0} y_{2}}{y_{1}}\right)_{*}=\left(\frac{-y_{0} y_{1}}{y_{2}}\right)_{*}=1
\end{array}\right\} .
$$

We next consider $c_{0}$, noting that $c_{0}=c_{1}=c_{2}$, by symmetry. If $y_{0}$ is even, we set $y_{0}=2 y_{0}^{\prime}$, where $y_{0}^{\prime}$ is odd. It then holds that

$$
\begin{aligned}
c_{0}= & \frac{1}{\pi} \cdot \sum_{\substack{\left(y_{0}^{\prime}, y_{1}, y_{2}\right) \in S_{-1} \\
y_{1} \equiv y_{2}(\bmod 8)}} \frac{2 \gamma\left(y_{0}^{\prime} y_{1} y_{2}\right)}{\left(2 y_{0}^{\prime} y_{1} y_{2}\right)^{\frac{3}{2}}} \\
& \times \prod_{p \mid y_{0}^{\prime}}\left(1+\left(\frac{y_{1} y_{2}}{p}\right)\right) \cdot \prod_{p \mid y_{1}}\left(1+\left(\frac{2 y_{0}^{\prime} y_{2}}{p}\right)\right) \cdot \prod_{p \mid y_{2}}\left(1+\left(\frac{-2 y_{0}^{\prime} y_{1}}{p}\right)\right) .
\end{aligned}
$$


Putting $d=y_{0}^{\prime} y_{1} y_{2}$ we deduce as above that

$$
c_{0}=\frac{1}{\pi} \cdot \sum_{\substack{d=1 \\ 2 \nmid d}}^{\infty} \frac{\mu^{2}(d) \cdot \gamma(d) \cdot 2^{\omega(d)}}{d^{\frac{3}{2}}} \cdot \frac{\Delta_{0}(d)}{\sqrt{2}},
$$

where now

$$
\Delta_{0}(d)=\#\left\{y_{0}^{\prime} y_{1} y_{2}=d: \begin{array}{l}
y_{1} \equiv y_{2}(\bmod 8), \\
\left(\frac{y_{1} y_{2}}{y_{0}^{\prime}}\right)_{*}=\left(\frac{2 y_{0}^{\prime} y_{2}}{y_{1}}\right)_{*}=\left(\frac{-2 y_{0}^{\prime} y_{1}}{y_{2}}\right)_{*}=1
\end{array}\right\}
$$

Bringing these expressions together in (13), we conclude that

$$
c=\frac{1}{\pi} \cdot \sum_{\substack{d=1 \\ 2 \nmid d}}^{\infty} \frac{\mu^{2}(d) \cdot \gamma(d) \cdot 2^{\omega(d)}}{d^{\frac{3}{2}}} \cdot\left(\Delta_{-1}(d)+\frac{3}{\sqrt{2}} \Delta_{0}(d)\right) .
$$

One finds by numerical computation that $c=2.68 \ldots$, as in Conjecture 1 .

\section{The lower bound}

Let $C \subset \mathbf{P}^{2}$ be a conic defined over $\mathbf{Q}$ and let $H: C(\mathbf{Q}) \rightarrow \mathbf{R}_{\geqslant 0}$ be an exponential height function. Suppose that $C$ is defined by a non-singular quadratic form defined over $\mathbf{Z}$ with relatively prime coefficients all bounded in modulus by $M$. A number of results in the literature are directed at estimating the counting function $N_{C, H}(P)=\#\{x \in C(\mathbf{Q}): H(x) \leqslant P\}$, as $P \rightarrow \infty$, with the outcome that there exist absolute constants $\delta, \psi>0$ such that

$$
N_{C, H}(P)=c_{H}\left(C\left(\mathbb{A}_{\mathbf{Q}}\right)\right) P+O\left(M^{\psi} P^{1-\delta}\right),
$$

where $c_{H}\left(C\left(\mathbb{A}_{\mathbf{Q}}\right)\right)$ is the constant predicted by Peyre [8]. This is a special case of the work of Franke, Manin and Tschinkel [5] on flag varieties $P \backslash G$, with $G$ taken to be the orthogonal group in three variables. Typically the uniformity in $M$ is not actually recorded, but it transpires that the dependence on $M$ is at worst polynomial.

We are now ready to establish Theorem 1 . For any choice of $\mathbf{y}$ there are clearly $O(1)$ rational points on $C_{\mathbf{y}}$ which correspond to a solution with $x_{0} x_{1} x_{2}=0$. Beginning with (2) we deduce that

$$
N_{1}(B) \geqslant \frac{1}{4} \sum_{\substack{\mathbf{y} \in \mathbf{N}^{3} \\ y_{0}, y_{1}, y_{2} \leqslant B^{\theta}}} \mu^{2}\left(y_{0} y_{1} y_{2}\right) N_{C_{\mathbf{y}}, H_{\mathbf{y}}}^{+}\left(B^{\frac{1}{2}}\right)+O\left(B^{3 \theta}\right)
$$

for any $\theta \leqslant \frac{1}{3}$, where $N_{C_{\mathbf{y}}, H_{\mathbf{y}}}^{+}$is defined as for $N_{C_{\mathbf{y}}, H_{\mathbf{y}}}$, but with the additional constraint that $\operatorname{gcd}\left(x_{0} y_{0}, x_{1} y_{1}, x_{2} y_{2}\right)=1$. Once taken in conjunction with the fact that $y_{0} y_{1} y_{2}$ is square-free and $\operatorname{gcd}\left(x_{0}, x_{1}, x_{2}\right)=1$, we see that the coprimality condition $\operatorname{gcd}\left(x_{0} y_{0}, x_{1} y_{1}, x_{2} y_{2}\right)=1$ on $C_{\mathbf{y}}$ is equivalent to demanding that $\operatorname{gcd}\left(x_{i}, x_{j}, y_{k}\right)=1$ for each permutation $\{i, j, k\}=\{0,1,2\}$. Using the Möbius function to remove these coprimality conditions gives

$$
N_{C_{\mathbf{y}}, H_{\mathbf{y}}}^{+}\left(B^{\frac{1}{2}}\right)=\sum_{k_{0} \mid y_{0}} \sum_{k_{1} \mid y_{1}} \sum_{k_{2} \mid y_{2}} \mu\left(k_{0} k_{1} k_{2}\right) N_{C_{\mathbf{k}, \mathbf{y}^{\prime}}, H_{\mathbf{k}, \mathbf{y}^{\prime}}}\left(\frac{B^{\frac{1}{2}}}{k_{0} k_{1} k_{2}}\right),
$$

where $y_{i}=k_{i} y_{i}^{\prime}$ for $0 \leqslant i \leqslant 2, C_{\mathbf{k}, \mathbf{y}^{\prime}}$ is the conic $k_{0} y_{0}^{\prime 3} x_{0}^{2}+k_{1} y_{1}^{\prime 3} x_{1}^{2}=k_{2} y_{2}^{\prime 3} x_{2}^{2}$ and $H_{\mathbf{k}, \mathbf{y}^{\prime}}$ is defined as for $H_{\mathbf{y}}$ but with $y_{i}^{3}$ replaced by $k_{i} y_{i}^{\prime 3}$, for $0 \leqslant i \leqslant 2$. The conic $C_{\mathbf{k}, \mathbf{y}^{\prime}}$ has an underlying quadratic form with coefficients of size at most $B^{3 \theta}$. Applying (15) we conclude that

$$
N_{C_{\mathbf{y}}, H_{\mathbf{y}}}^{+}\left(B^{\frac{1}{2}}\right)=B^{\frac{1}{2}} \sum_{k_{0} \mid y_{0}} \sum_{k_{1} \mid y_{1}} \sum_{k_{2} \mid y_{2}} \frac{\mu\left(k_{0} k_{1} k_{2}\right)}{k_{0} k_{1} k_{2}} \cdot c_{H_{\mathbf{k}, \mathbf{y}^{\prime}}}\left(C_{\mathbf{k}, \mathbf{y}^{\prime}}\left(\mathbb{A}_{\mathbf{Q}}\right)\right)+O_{\varepsilon}\left(B^{\frac{1-\delta}{2}+3 \theta \psi+\varepsilon}\right),
$$


for any $\varepsilon>0$. One finds that the main term here is precisely equal to $c_{H_{\mathbf{y}}}\left(C_{\mathbf{y}}\left(\mathbb{A}_{\mathbf{Q}}\right)^{+}\right) B^{\frac{1}{2}}$, in the notation of $\S 2$. Noting that

$$
\sum_{y \leqslant B^{\theta}} \frac{f(y)}{y^{\frac{3}{2}}}=\sum_{y=1}^{\infty} \frac{f(y)}{y^{\frac{3}{2}}}+O\left(B^{-\frac{\theta}{2}+\varepsilon}\right)
$$

for any arithmetic function $f$ satisfying $f(n)=O_{\varepsilon}\left(n^{\varepsilon}\right)$, we deduce that

$$
N_{1}(B) \geqslant c B^{\frac{1}{2}}+O\left(B^{3 \theta}\right)+O_{\varepsilon}\left(B^{\frac{1-\delta}{2}+3 \theta(1+\psi)+\varepsilon}\right)+O_{\varepsilon}\left(B^{\frac{1-\theta}{2}+\varepsilon}\right),
$$

for any $\varepsilon>0$. We therefore conclude the proof of Theorem 1 by taking $\theta$ to satisfy the inequalities $0<\theta<\frac{\delta}{6(1+\psi)}$.

\section{The upper bound}

The aim of this section is to prove Theorem 2, for which our starting point is (1). In order to estimate $N_{1}(B)$ we will view the equation in two basic ways: either as a family of conics or as a family of plane cubic curves. The work of Heath-Brown [6] allows one to estimate rational points of bounded height on plane curves, uniformly in the coefficients of the underlying equation. We will invoke this theory through the prism of the first author's work [2, Lemma 4.10], which yields the following bound for any integer $d \geqslant 2$.

Lemma 3. Let $\mathbf{c} \in \mathbf{Z}^{3}$ with $c_{1} c_{2} c_{3} \neq 0$ and pairwise coprime coordinates. Then we have

$$
\#\left\{\mathbf{z} \in \mathbf{Z}^{3}: \begin{array}{l}
\operatorname{gcd}\left(z_{1}, z_{2}, z_{3}\right)=1,\left|z_{i}\right| \leqslant Z_{i}, \\
c_{1} z_{1}^{d}+c_{2} z_{2}^{d}+c_{3} z_{3}^{d}=0
\end{array}\right\} \ll_{d}\left(1+\frac{Z_{1} Z_{2} Z_{3}}{\left|c_{1} c_{2} c_{3}\right|^{\frac{2}{d}}}\right)^{\frac{1}{3}} d^{\omega\left(c_{1} c_{2} c_{3}\right)} .
$$

We will also make use of the familiar bound $\sum_{n \leqslant x} k^{\omega(n)} \ll x \log ^{k-1} x$, which is valid for any $k \in \mathbf{N}$. We consider the contribution $N(\mathbf{X}, \mathbf{Y})$, say, to $N_{1}(B)$ from $\mathbf{x}, \mathbf{y}$ such that

$$
X_{i} \leqslant x_{i}<2 X_{i}, \quad Y_{i} \leqslant y_{i}<2 Y_{i}
$$

for $0 \leqslant i \leqslant 2$. Clearly $N(\mathbf{X}, \mathbf{Y})=0$ unless $X_{i}^{2} Y_{i}^{3} \leqslant B$ and $X_{i}, Y_{i}>1 / 2$, for $0 \leqslant i \leqslant 2$. It will be convenient to set $X=X_{0} X_{1} X_{2}$ and $Y=Y_{0} Y_{1} Y_{2}$. In particular we may henceforth assume that $X^{2} Y^{3} \leqslant B^{3}$. On summing over dyadic intervals we see that

$$
N_{1}(B) \ll \log ^{6} B \max _{\mathbf{X}, \mathbf{Y}} N(\mathbf{X}, \mathbf{Y})
$$

where the maximum is over $\mathbf{X}, \mathbf{Y}$ satisfying the above inequalities.

Viewing the underlying equation as a family of conics first, we take $d=2$ in Lemma 3 and deduce that

$$
\begin{aligned}
N(\mathbf{X}, \mathbf{Y}) & \ll \sum_{\mathbf{y}} 2^{\omega\left(y_{0} y_{1} y_{2}\right)}\left(1+\frac{X}{Y^{3}}\right)^{\frac{1}{3}} \\
& \ll\left(Y+X^{\frac{1}{3}}\right) \log ^{3} B .
\end{aligned}
$$

Alternatively, regarding the equation as a family of cubics, we take $d=3$ in Lemma 3 and obtain

$$
\begin{aligned}
N(\mathbf{X}, \mathbf{Y}) & \ll \sum_{\mathbf{x}} 3^{\omega\left(x_{0} x_{1} x_{2}\right)}\left(1+\frac{Y}{X^{\frac{4}{3}}}\right)^{\frac{1}{3}} \\
& \ll\left(X+Y^{\frac{1}{3}} X^{\frac{5}{9}}\right) \log ^{6} B .
\end{aligned}
$$

Bringing these two estimates together we conclude that

$$
N(\mathbf{X}, \mathbf{Y}) \ll\left(\min \{X, Y\}+\min \left\{Y, Y^{\frac{1}{3}} X^{\frac{5}{9}}\right\}+X^{\frac{1}{3}}\right) \log ^{6} B
$$

Now it is clear that $\min \{X, Y\} \leqslant X^{\frac{2}{5}} Y^{\frac{3}{5}} \leqslant B^{\frac{3}{5}}$ and

$$
\min \left\{Y, Y^{\frac{1}{3}} X^{\frac{5}{9}}\right\} \leqslant Y^{\frac{9}{25}} \cdot\left(Y^{\frac{1}{3}} X^{\frac{5}{9}}\right)^{\frac{18}{25}}=X^{\frac{2}{5}} Y^{\frac{3}{5}} \leqslant B^{\frac{3}{5}},
$$


since $X^{2} Y^{3} \leqslant B^{3}$. Finally we note that $X^{\frac{1}{3}} \leqslant B^{\frac{1}{2}}$. Inserting our estimate for $N(\mathbf{X}, \mathbf{Y})$ into (16), we therefore arrive at the statement of Theorem 2 .

\section{References}

[1] D. Abramovich, Birational geometry for number theorists. Arithmetic geometry, 335-373, Amer. Math. Soc., 2009.

[2] T.D. Browning, Quantitative arithmetic of projective varieties. Progress in Math. 277, Birkhäuser, 2009.

[3] F. Campana, Fibres multiples sur les surfaces: aspects geométriques, hyperboliques et arithmétiques. Manuscripta Math. 117 (2005), 429-461.

[4] P. Erdős and G. Szekeres, Über die Anzahl der Abelschen Gruppen gegebener Ordnung und über ein verwandtes zahlentheoretisces Problem. Acta Univ. Szeged 7 (1934-1935), 95-102.

[5] J. Franke, Y.I. Manin and Y. Tschinkel, Rational points of bounded height on Fano varieties. Invent. Math. 95 (1989), 421-435.

[6] D.R. Heath-Brown, The density of rational points on curves and surfaces. Annals of Math. 155 (2002), 553-595.

[7] A. Nitaj, On a conjecture of Erdős on 3-powerful numbers. Bull. London Math. Soc. 27 (1995), 317318.

[8] E. Peyre, Hauteurs et nombres de Tamagawa sur les variétés de Fano. Duke Math. J. 79 (1995), 101-218.

[9] E. Peyre and Y. Tschinkel, Tamagawa numbers of diagonal cubic surfaces, numerical evidence. Math. Comp. 70 (2001), 367-387.

[10] B. Poonen, The projective line minus three fractional points. http://www-math.mit.edu/ poonen/ slides/campana_s.pdf, July 2006.

[11] K. Van Valckenborgh, Squareful numbers in hyperplanes. Submitted, 2010 (arXiv:1001.3296).

T.D. Browning

School of Mathematics

University of Bristol

Bristol

BS8 1TW

United Kingdom

e-mail: t.d.browning@bristol.ac.uk

K. Van Valckenborgh

Department of Mathematics

K.U. Leuven

Celestijnenlaan 200B

3001 Leuven

Belgium

e-mail: karl . vanvalckenborgh@wis. kuleuven. be 